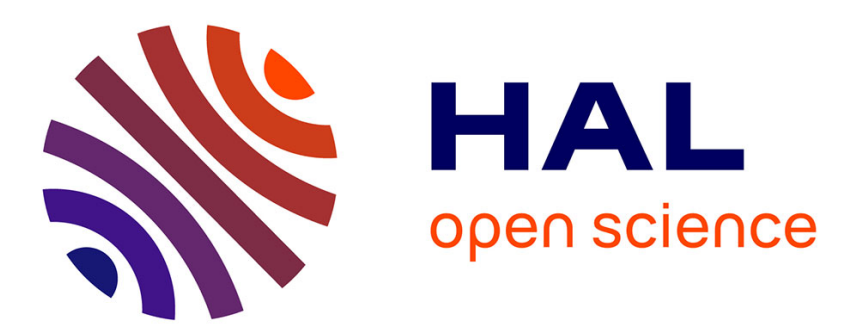

\title{
Radon transforms on generalized Cormack's curves and a new Compton scatter tomography modality
}

\author{
T. T. Truong, M. K. Nguyen
}

\section{To cite this version:}

T. T. Truong, M. K. Nguyen. Radon transforms on generalized Cormack's curves and a new Compton scatter tomography modality. Inverse Problems, 2011, 27 (12), pp.125001. 10.1088/0266$5611 / 27 / 12 / 125001$. hal-00670837

\section{HAL Id: hal-00670837 \\ https://hal.science/hal-00670837}

Submitted on 16 Feb 2012

HAL is a multi-disciplinary open access archive for the deposit and dissemination of scientific research documents, whether they are published or not. The documents may come from teaching and research institutions in France or abroad, or from public or private research centers.
L'archive ouverte pluridisciplinaire HAL, est destinée au dépôt et à la diffusion de documents scientifiques de niveau recherche, publiés ou non, émanant des établissements d'enseignement et de recherche français ou étrangers, des laboratoires publics ou privés. 


\title{
Radon transforms on generalized Cormack's curves and a new Compton scatter tomography modality
}

\author{
T. T. Truong $\dagger \dagger$ and M. K. Nguyen $\ddagger$ \\ † Laboratoire de Physique Théorique et Modélisation \\ (CNRS UMR 8089/Université de Cergy-Pontoise), \\ 2 av. Adolphe Chauvin 95302 Cergy-Pontoise, France. \\ $\ddagger$ Laboratoire Equipes Traitement de l'Information et Systèmes, \\ (CNRS UMR 8051/ENSEA/Université de Cergy-Pontoise), \\ 2 av. Adolphe Chauvin 95302 Cergy-Pontoise, France.
}

\begin{abstract}
In his seminal work of 1981, A M Cormack has established that Radon transforms defined on two remarkable families of curves in the plane are invertible and admit explicit inversion formulas via circular harmonic decomposition. A sufficient condition for finding larger classes of curves enjoying the same property is given in this paper. We show that these generalized Cormack's curves are given by the solutions of a non-linear first-order differential equation, which is invariant under geometric inversion. A derivation of the analytic inverse formula of the corresponding Radon transforms, as well as some of their main properties, are worked out. Interestingly, among these generalized Cormack's curves are circles orthogonal to a circle of fixed radius centered at the origin of coordinates. It is suggested that a novel Compton Scatter Tomography (CST) modality may be modeled by a Radon transform defined on these circles.
\end{abstract}

\section{Introduction}

Ever since the Radon transform has appeared as a key working tool in imaging science, there is a continuing interest to extend it to non-trivial curves in the plane for which the transform remains invertible and for which an explicit inversion formula can be written out. It was A M Cormack, while working on the inversion of the classical Radon transform, who first found that the Radon transform on circles intersecting a fixed point in the plane enjoys such inversion properties [1]. Later in 1981 he found two new families of curves, for which the related Radon transforms display precisely the same inversion properties $[2,3]$. In the past decades, progress has been achieved also in the inversion of Radon transforms on circles centered on a line (resp. on another circle) in the context of Synthetic Aperture Radar imaging [4, 5] (resp. in thermoacoustic tomography [6]). At the beginning of the 90's, A Kurusa has attempted to generalize the Cormack's result to curves of abstract two-dimensional manifolds equipped with a metric and a distance function $[7,8]$. In particular he established invertibility for Radon transforms on curves having strictly convex distance function in two-point homogeneous spaces and spaces of constant curvature. However no concrete

$†$ To whom correspondence should be addressed (truong@u-cergy.fr) 
curve was given explicitly. Thus it would be of interest, to search for other classes of curves in $\mathbb{R}^{2}$ for which the inversion problem of the corresponding Radon transforms can be solved in the same way.

Amid a wealth of general results on Radon transform on curves in the plane $[9,10,11,12]$, we shall be concerned here with Radon transforms on families of curves which are invariant under a global rotation around the origin of a polar coordinate system $O$ and which have an axis of reflection symmetry intersecting $O$. In this situation, it is appropriate to use the so-called circular harmonic component decomposition of functions (see e.g. [13, 14]), which helps to reformulate the Radon transform as an integral transform of Tchebyscheff's type, as appearing e.g. in [15].

In section 2 of this paper, we consider a generalization of Cormack's curves, which preserves their symmetry and smoothness properties and for which the corresponding Radon transform is invertible in the setting of the circular harmonic component formalism. It turns out that demanding that the integral equation for circular harmonic components be invertible implies solving a differential equation of first order. The solutions of this equation describes three classes of curves. Each one is given by a function of $r^{\sigma}$, where $\sigma \in(\mathbb{R} \backslash\{0\})$ is a parameter. The simplest representative of each group is clearly labeled by $|\sigma|=1$, whereas any other member of this group may viewed as its $\sigma$-descendant. The first group, which corresponds to the simplest structure, is precisely the one found by A M Cormack in 1981, of which the two simplest representatives are the straight line $(\sigma=1)$ and the circle through the origin $\sigma=-1$. For the second group the simplest representatives are the circular arcs subtended by a common chord of fixed length which rotates freely around its middle point. The Radon problem for this class of circular arcs has been shown to be soluble in [16]. The third and last group is new and its simplest representatives are circular arcs which intersect orthogonally a fixed circle. A detailed study of this class of new Radon transform is given in section 3. A particular emphasis is put on the derivation of its analytic inverse formula and its consequences. This last result is interesting in so far as it suggests a new operating modality for Compton Scatter Tomography (CST), a much investigated ionizing radiation imaging process nowadays. This shall be presented in section 4 . Finally a conclusion closes the text with some promising research topics.

\section{Generalized Cormack's curves and the associated Radon transforms}

\subsection{Cormack's curves and the related Radon's problem}

A Cormack's curve $\mathcal{C}_{\sigma}$ is characterized by two parameters: $\phi$, an angle specifying its rotational state around the coordinate origin and $p$, a length which describes its "size". In a polar coordinate system $(r, \theta)$ let

$$
\cos \sigma(\theta-\phi)=\left(\frac{p}{r}\right)^{\sigma}
$$

be its equation, where $\sigma \in\{\mathbb{R} \backslash\{0\}\}$ and $|\theta-\phi|<\pi / 2 \sigma$. $\mathcal{C}_{\sigma}$ has an obvious axis of reflection symmetry (labeled by $\phi$ ) which goes through the coordinate origin $O$. We have chosen this form but could have also taken $r / p$ instead of $p / r$. This does not affect the generality of the argumentation. $\sigma>0$ (resp. $\sigma<0$ ) corresponds to the $\alpha$ $(\beta-)$ curves of Cormack in $[2]$.

The $\alpha$-curves (or $\sigma>0$-curves) tend to infinity as $|\theta-\phi| \rightarrow \pi / 2 \alpha$; they intersect themselves at least once if $0<\alpha<1 / 2$, but they do not intersect themselves if 
$\alpha \geq 1 / 2$. For $\alpha=1 / 2,1,2$ we have the parabola, the straight line and the onebranch hyperbola. The $\beta$-curves (or $\sigma<0$ curves) tend to the coordinate origin as $|\theta-\phi| \rightarrow \pi / 2 \beta$; they intersect themselves at least once if $0<\beta<1 / 2$, but they do not intersect themselves if $\beta \geq 1 / 2$. For $\beta=1 / 2,1,2$ we have the cardioid, the circle through the coordinate origin and the one-branch lemniscate of Bernouilli. $\alpha$ - and $\beta$ curves are exchanged under geometric inversion in the unit circle $(r, \theta) \rightarrow(1 / r, \theta)$.

The Radon transform on these curves $\mathcal{C}_{\sigma}$ maps a real-valued integrable function $f(r, \theta)$ on $\mathbb{R}^{2}$ onto its integral on a curve $\mathcal{C}_{\sigma}$, i.e.

$$
\widehat{f}(p, \phi)=\int_{(r, \theta) \in \mathcal{C}_{\sigma}} d l_{\sigma} f(r, \theta),
$$

where $d l_{\sigma}$ is the line element of $\mathcal{C}_{\sigma}$.

Let $f(r, \theta) \in L^{1}\left(\mathbb{R}_{+} \times \mathbb{S}^{1}\right)$, continuous and bounded. Then $f(r, \theta)$ admits the following circular harmonic expansion

$$
f(r, \theta)=\sum_{l=-\infty}^{\infty} f_{l}(r) e^{i l \theta}, \quad \text { with } \quad f_{l}(r)=\frac{1}{2 \pi} \int_{0}^{2 \pi} d \theta e^{-i l \theta} f(r, \theta) .
$$

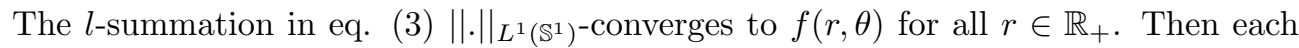
$f_{l}(r)$ is bounded and continuous on $\mathbb{R}_{+}$.

Substitution of (3) into eq. (2) shows that $\widehat{f}(p, \phi)$ is of the form

$$
\widehat{f}(p, \phi)=\sum_{l \in \mathbb{Z}} \widehat{f}_{l}(p) \mathrm{e}^{i l \phi}, \quad \text { where } \quad \widehat{f}_{l}(r)=\frac{1}{2 \pi} \int_{0}^{2 \pi} d \theta e^{-i l \theta} \widehat{f}(\tau, \phi) .
$$

Then $\widehat{f}_{l}(p)$ emerges as a Tchebyscheff integral transform of $f_{l}(r)$. A M Cormack has showed that it can be inverted using the value of a special integral on a product of Tchebyscheff polynomials $[2,17]$.

So it is natural to raise the question whether there exists other classes of curves that lend themselves to the same inversion technique.

\subsection{A generalization of Cormack's curves}

An extended form of Cormack's curves, which would display the same symmetry, but leave the size and the shape arbitrary, is expected to have the following properties:

- These curves may have an equation of the form

$$
\cos \sigma(\theta-\phi)=\frac{g\left(r^{\sigma}\right)}{h(p)}
$$

where $g(x)$ and $h(x)$ are real-valued and sufficiently smooth functions. (A more general implicit relation of the type $\cos \sigma(\theta-\phi)=G_{p}(r)$ or even $F_{p}(\cos \sigma(\theta-$ $\phi), r)=0$ could be envisaged but would lead to unmanageable complications). As $p$ is an arbitrary length scale, we may set $\tau=h(p)>0$ for simplicity (since its sign can be absorbed in $g(x))$. From now on, we call a generalized Cormack curve $\mathcal{C}(\tau, \phi)$, the curve of equation

$$
\cos \sigma \gamma=\frac{g\left(r^{\sigma}\right)}{\tau}
$$

where $\gamma=(\theta-\phi)$. 
- The reality of the curve requires that $\left|g\left(r^{\sigma}\right)\right| / \tau<1$, and for $0<\sigma \gamma<\pi$, one may write

$$
\gamma=\frac{1}{\sigma} \cos ^{-1}\left(\frac{g\left(r^{\sigma}\right)}{\tau}\right)
$$

- Conversely assuming that the inverse function $g^{-1}$ of $g$ exists (with possibly a restriction on the range of $\tau)$, one can solve for $r(\gamma)=\left(g^{-1}(\tau \cos \sigma \gamma)\right)^{1 / \sigma}$. This means that there may be several branches $r(\gamma)$.

- Each branch may or may not be of finite extent but is always expected to be of smooth tracing on both sides of the symmetry axis. Let $\zeta=\min _{r \in \mathbb{R}_{+}} \mid(g(r) \mid$. Eq. (5) shows that this curve branch exists if $\zeta<\tau$ and that it is situated in an angular sector $-\gamma_{0}<\gamma<\gamma_{0}$, with $\gamma_{0}$ given by $\cos \sigma \gamma_{0}= \pm \zeta / \tau$. In this case one can picture this branch as starting from $\gamma=0$ at a distance $r=r_{0}$ on the symmetry axis, $\left(r_{0}\right.$ being the solution of $\left.\tau=g\left(r_{0}^{\sigma}\right)\right)$ and ending at the point of polar coordinates $\left(r_{0}^{*}, \gamma_{0}\right) \quad\left(r_{0}^{*}\right.$ being the solution of $\pm \zeta=g\left(\left(r_{0}^{*}\right)^{\sigma}\right)$ ).

To stress the special character of these curves, we cite two classes of curves which are not $\sigma$-generalized Cormack's curves. They are given by the following equations:

$$
\cos \sigma \gamma=\left(r^{\sigma}+\frac{p(2 R+p)}{r^{\sigma}}\right) \frac{1}{2(p+R)} \quad \text { and } \quad \cos \sigma \gamma=\left(r^{\sigma}+\frac{p^{2}-R^{2}}{r^{\sigma}}\right) \frac{1}{2 p} .
$$

There is no factorization of the right-hand-side into a product of a function of $\tau=h(R)$ and a function $g\left(r^{\sigma}\right)$. For $\sigma=1$, they represent two families of circles of variable radius $R$, which are respectively externally tangent or centered on a fixed circle of radius $p$ and with center $O$, the origin of polar coordinates.

\subsection{The Radon transform on generalized Cormack's curves $\mathcal{C}(\tau, \phi)$}

Physical quantities (such as mass density, radio-activity density or linear attenuation coefficient, etc.), which are relevant for imaging processes, are generally real-valued, non-negative, smooth and compactly supported functions of polar coordinates $(r, \theta)$. However we shall consider, from now on, as A M Cormack did in [2], functions $f(r, \theta)$ which are, with respect to variables $x=r \cos \theta$ and $y=r \sin \theta$, in Schwartz space $\mathcal{S}\left(\mathbb{R}^{2}\right)$ of the infinitely smooth functions (i.e. decaying faster than any power of $x$ and $y$ at infinity as well as their derivatives). Then it can be checked that $f_{l}(r) \in \mathcal{S}\left(\mathbb{R}_{+}\right)$.

Definition 2.1 The Radon transform $\widehat{f}(\tau, \phi)$ of function $f(r, \theta) \in \mathcal{S}\left(\mathbb{R}^{2}\right)$, on a specific branch of a generalized Cormack's curve $\mathcal{C}(\tau, \phi)$, is given by

$$
\widehat{f}(\tau, \phi)=\int_{(r, \theta) \in \mathcal{C}(\tau, \phi)} d l f(r, \theta),
$$

where, for simplicity we designate the chosen curve branch by the same symbol $\mathcal{C}(\tau, \phi)$ and $d l$ its arc element. $d l$ can be computed from eq. (5), i.e.

$$
d l=d \gamma\left\{r \sqrt{1+\frac{\tau^{2}-g^{2}\left(r^{\sigma}\right)}{r^{2 \sigma} g^{\prime 2}\left(r^{\sigma}\right)}}\right\}_{r=\left(g^{-1}(\tau \cos \sigma \gamma)\right)^{1 / \sigma}}=d r \sqrt{\frac{\tau^{2}-g^{2}+\left(r^{\sigma} g^{\prime}\right)^{2}}{\tau^{2}-g^{2}}},
$$

$g^{\prime}(x)$ being the derivative of $g(x)$. 
Radon transforms on generalized Cormack's curves and a new Compton scatter tomography modality5

Eq. (8) may now be given explicitly as an integral on $\gamma$

$$
\widehat{f}(\tau, \phi)=\int_{-\gamma_{0}}^{\gamma_{0}} d \gamma\left\{\sqrt{\frac{\tau^{2}-g^{2}+\left(r^{\sigma} g^{\prime}\right)^{2}}{\left(r^{\sigma} g^{\prime}\right)^{2}}}(r f(r, \gamma+\phi))\right\}_{r=\left(g^{-1}(\tau \cos \sigma \gamma)\right)^{1 / \sigma}},
$$

or as an integral on $r$

$\widehat{f}(\tau, \phi)=\left.\int_{r_{0}^{*}}^{r_{0}} d r \sqrt{\frac{\tau^{2}-g^{2}+\left(r^{\sigma} g^{\prime}\right)^{2}}{\tau^{2}-g^{2}}}(f(r, \gamma+\phi)+f(r,-\gamma+\phi))\right|_{\gamma=\frac{1}{\sigma} \cos ^{-1}\left(\frac{g(r \sigma)}{\tau}\right)}$,

where $\gamma_{0}, r_{0}$ and $r_{0}^{*}$ have been specified in subsection 2.2. As the singularities of the integrand, either in $\gamma$ or in $r$ are integrable, $\widehat{f}(\tau, \phi)$ is well defined and has the following elementary properties:

- $\widehat{f}(\tau, \phi)$ is a smooth and strongly decreasing function of $\tau(0<\zeta<\tau)$,

- for non-negative $f(r, \theta), \widehat{f}(\tau, \phi)$ is non-negative,

- the support of $\widehat{f}$ is generally not compact since $\zeta<\tau<\infty$ and $\phi \in[0,2 \pi]$.

Proposition 2.2 Under the above assumptions, $\widehat{f}(\tau, \phi)$ is defined for all pairs $(\tau, \phi)$ with $(0<\zeta<\tau<\infty$ and $0<\phi<2 \pi)$ and fully determined by its circular harmonic components $\widehat{f}_{l}(\tau)$, which are given by

$$
\widehat{f}_{l}(\tau)=2 \int_{r_{0}}^{r_{0}^{*}} d r \sqrt{\frac{\tau^{2}-g^{2}+\left(r^{\sigma} g^{\prime}\right)^{2}}{\tau^{2}-g^{2}}} \cos \left(\frac{l}{\sigma} \cos ^{-1} \frac{g}{\tau}\right) f_{l}(r),
$$

where the integration boundary $r_{0}$ and $r_{0}^{*}$ are given in subsection 2.2 .

Proof: In eq. (10), we insert the circular harmonic expansion (3) of $f(r, \theta)$. Then using $\gamma=(\theta-\phi)$, we rewrite the result as a circular harmonic expansion with respect to the angle $\phi$, so as to identify the $\widehat{f}_{l}(\tau)$ circular harmonic component of $\widehat{f}(\tau, \phi)$ as an integral of $f_{l}(r)$ over $\gamma$. Next we take into account the fact that beside the factor $e^{i l \gamma}$, the rest of the integrand is a function of $\cos \sigma \gamma$ and that the integration range is $\left[-\gamma_{0}, \gamma_{0}\right]$. Thus we can reduce it to $\left[0, \gamma_{0}\right]$ but insert of a global factor 2 . We now change the integration variable from $\gamma$ to $r$ and using eq. (9) we obtain eq. (12).

Eq. (12) displays the appropriate features required for discussing Cormack's inversion procedure [2], in the coming subsection.

2.4. A sufficient condition on the curves $\mathcal{C}(\tau, \phi)$ for the invertibility of the corresponding Radon tranform

Inspection shows that by imposing an appropriate condition on the structure of eq. (12) and by redefining its input and output functions, this equation will take the form of the Radon transform on Cormack's curves $\mathcal{C}_{\sigma}$. This fact can be formulated as

Theorem 2.3 Let $g$ be chosen so as to satisfy the condition

$$
-g^{2}\left(r^{\sigma}\right)+\left(r^{\sigma} g^{\prime}\left(r^{\sigma}\right)\right)^{2}=C,
$$

where $C$ is a constant. Define

$$
\widehat{F}_{l}(\tau)=\frac{\tau \widehat{f}_{l}(\tau)}{\sqrt{\tau^{2}+C}} \quad \text { and } \quad F_{l}(g)=\frac{1}{d g / d r} f_{l}\left(\left(g^{-1}\right)^{\frac{1}{\sigma}}\right)
$$


Radon transforms on generalized Cormack's curves and a new Compton scatter tomography modality6 then $\widehat{F}_{l}(\tau)$ and $F_{l}(g)$ are related by

$$
\widehat{F}_{l}(\tau)=2 \int_{\zeta \tau}^{\tau} d g \frac{\cos \left(\frac{l}{\sigma} \cos ^{-1} \frac{g}{\tau}\right)}{\sqrt{1-\left(\frac{g}{\tau}\right)^{2}}} F_{l}(g),
$$

where $\zeta$ has been defined in subsection (2.2).

Eq. (15) has precisely the form of the integral relation for circular components in the Radon problem on $\beta$-curves of [2] i.e. $\left(\beta=\sigma^{-1}\right)$, except perhaps for a non-zero lower integration bound $\ddagger$.

Proof: Eq. (15) can be established by taking into account condition (13) and the definitions (14) in eq. (12), in which one makes a further change of variable from $r$ to $g$. In [2], for the Radon problem on the $\beta$-curves, A M Cormack has obtained an equation linking the circular components of the function and its Radon transform, which has the same form as eq. (15), except a zero as lower integration bound instead of $\zeta \tau$.

Remark 2.4 Instead of using the variables $(g, \tau)$, we could have used $(1 / g, 1 / \tau)$. This would lead to an integral transform for the Radon problem on Cormack's $\alpha$-curves, see eq. (12a) in [2] modulo an integration bound. The connection between the two situations can be established by geometric inversion, as discussed in [2, 18].

Consequently we have

Corollary 2.5 The Radon transform on generalized Cormack curves $\mathcal{C}(\tau, \phi)$, defined by eq. (13), can be inverted by inverting the integral equation for their circular components (15), following A M Cormack's method in [2].

We shall discuss concrete cases of inversion, according to the nature of the solutions of eq. (13), in the following sections.

\subsection{Solutions of the differential equation determining the generalized Cormack's curves $\mathcal{C}(\tau, \phi)$}

Thus it becomes highly interesting to know what are the generalized Cormack's curves. To find them we have to solve eq. (13), a non-linear separable differential equation of first order, which takes a simple form in the variable $x=r^{\sigma}$

$$
\frac{d g}{\sqrt{g^{2}+C}}= \pm \frac{d x}{x} .
$$

There are three families of solutions according to the values of $C$ and dependent on an integration constant $s$.

- a) For $C=0$, the solution is trivial and yields the Cormack's curves,

$$
g(x)=\left(\frac{s}{x}\right) \quad \text { or } \quad g(x)=\left(\frac{x}{s}\right) .
$$

Here $s$ is an integration constant. If we set $s=p^{\sigma}$ in eq. (17) we find the equations of the $\alpha$ - and $\beta$-Cormack's curves of [2]

$$
\cos \sigma \gamma=\left(\frac{p}{r}\right)^{\sigma} \quad \text { or } \quad \cos \sigma \gamma=\left(\frac{r}{p}\right)^{\sigma} \text {. }
$$

$\ddagger$ Note that this form has been obtained also by A Kurusa in his work on Radon transform on a half-sphere [19]. 
- b) For $C=b^{2}>0$ with $b \in \mathbb{R}$, we have the solution

$$
g(x)= \pm \frac{|b|}{2}\left(\frac{s}{x}-\frac{x}{s}\right)
$$

In what follows $|b|$ can be absorbed in the definition of $\tau$. The $(-)$ sign in $g$ may be absorbed by an angular shift in $\gamma \rightarrow(\gamma \pm \pi / 2 \sigma)$ without changing the nature of the curve branch. Hence it is sufficient to consider curves of equation

$$
\cos \sigma \gamma=\frac{1}{2 \tau}\left[\left(\frac{p}{r}\right)^{\sigma}-\left(\frac{r}{p}\right)^{\sigma}\right] .
$$

The Radon transform on such curves with $\sigma=1$, as well as two special cases with $\sigma=1 / 2,2$, has been discussed in [16].

- c) For $C=-b^{2}>0$ with $b \in \mathbb{R}$, we obtain

$$
g(x)= \pm \frac{|b|}{2}\left(\frac{s}{x}+\frac{x}{s}\right)
$$

Following the arguments given in b), without loss of generality we can restrict ourselves to curves given by

$$
\cos \sigma \gamma=\frac{1}{2 \tau}\left[\left(\frac{p}{r}\right)^{\sigma}+\left(\frac{r}{p}\right)^{\sigma}\right] .
$$

For the three classes, note that when $\sigma=1 / m$, with $m=1,2,3, \ldots$, equations $(17,19,20)$ refer to higher order algebraic curves of special types. In particular for $\sigma=1$ the corresponding curves are a straight line and three families of circles or arcs of circle in the plane (see Fig. 2). Line and circles in a) and b) are associated to

- the usual Radon transform on straight lines, which is widely used in Computed Tomography (CT), Single Photon Emission Computed Tomography (SPECT) and Positron Emission Tomography (PET),

- the Radon transform on circles passing through a fixed point, used in S J Norton's Compton Scatter Tomography (CST) modality [21],

- the Radon transform on rotating arcs of circle, arising in a recent CST modality $[16]$.

The most valuable property one can expect from a Radon transform is, without any doubt, its invertibility since it solves in principle the inverse problem associated to some imaging processes, as in three cases of the $\sigma=1$ curves above. The last family of circles, which correspond to $C<0$, has not yet been considered in the literature and will be the subject of section 3 .

\subsection{Inversion of Radon transforms on generalized Cormack's curves with $C \geqslant 0$}

The case with $C=0$ shall not be discussed in this paper since it has appeared in the work of A M Cormack [2].

Let us consider now the case with $\sigma>0$ and $C>0$, with the provisions b) of subsection 2.5. In [16], we have used a different inversion approach and put the emphasis on the special case $\sigma=1$ in view of an application in Compton Scatter Tomography (CST). Here we follow the general approach presented above. The lower bound in the integral of eq. (15) is 0 , since for $r=p$, we have $\zeta_{0}=0$ and consequently $\gamma_{0}=\pi / 2 \sigma$. Thus eq. (15) becomes identical to the equation for the Radon transform on Cormack's $\beta$-curves [2]. 
Radon transforms on generalized Cormack's curves and a new Compton scatter tomography modality 8

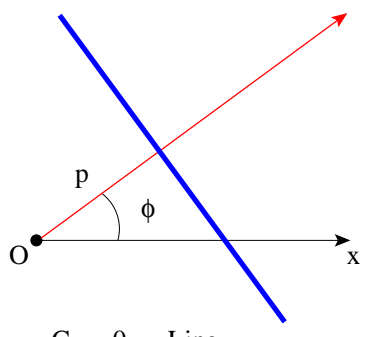

$\mathrm{C}=0 \quad$ Line

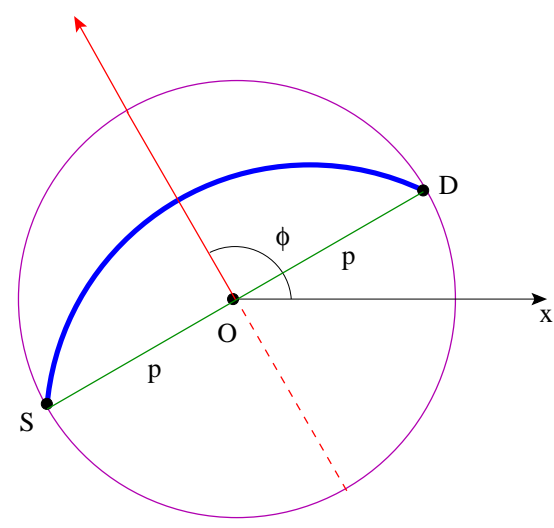

C $>0$ Circular arc

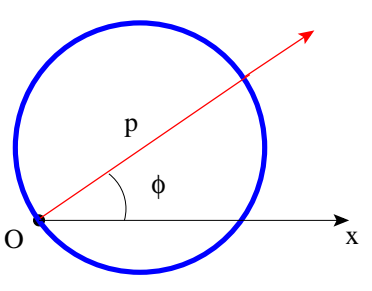

$\mathrm{C}=0$ Circle through 0

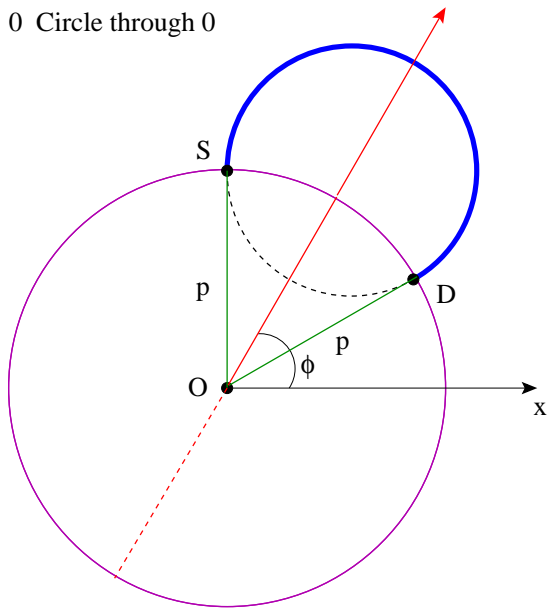

$\mathrm{C}<0 \quad$ Circular arc

Figure 1. Representations of the $\sigma=1$ solutions of eq. (16)

Let $\widehat{f}(\tau, \phi)$ be the Radon transform of a $f(r, \theta) \in \mathcal{S}\left(\mathbb{R}^{2}\right)$ on the generalized Cormack's curve of equation

$$
\cos \sigma \gamma=\frac{1}{2 \tau}\left[\left(\frac{p}{r}\right)^{\sigma}-\left(\frac{r}{p}\right)^{\sigma}\right] .
$$

Proposition 2.3 shows that via the change of functions

$$
\widehat{F}_{l}(\tau)=\frac{\tau \widehat{f}_{l}(\tau)}{\sqrt{1+\tau^{2}}} \quad \text { and } \quad F_{l}(g)=\left(-\frac{p}{\sigma}\right) \frac{f_{l}\left(p\left(\sqrt{g^{2}+1}-g\right)^{1 / \sigma}\right)}{\sqrt{g^{2}+1}\left(\sqrt{g^{2}+1}+g\right)^{\frac{1}{\sigma}}},
$$

where $f_{l}(r)$ (resp. $\widehat{f}_{l}(\tau)$ ) are the circular harmonic components of $f(r, \theta)$ (resp. $\widehat{f}(\tau, \phi))$, eq. (15) becomes precisely the integral transform for the circular components of functions in the Radon problem on Cormack's $\beta$-curves (see his eq. (12b) in [2]):

$$
\widehat{F}_{l}(\tau)=2 \int_{0}^{\tau} d g \frac{\cos \left(\frac{l}{\sigma} \cos ^{-1} \frac{g}{\tau}\right)}{\sqrt{1-\left(\frac{g}{\tau}\right)^{2}}} F_{l}(g) .
$$

Observe that $\widehat{F}_{l}(\tau)$ (resp. $\left.F_{l}(g)\right)$ enjoy the same smoothness properties as $\widehat{f}_{l}(\tau)$ (resp. $\left.f_{l}(r)\right)$. As A M Cormack has worked out fully the inversion procedure in $[2,22]$, we can state the following theorem 
Radon transforms on generalized Cormack's curves and a new Compton scatter tomography modality9

Theorem 2.6 The Radon transform of functions $f(r, \theta) \in \mathcal{S}\left(\mathbb{R}^{2}\right)$ on the generalized Cormack's curves of equation

$$
\cos \sigma \gamma=\frac{g(r)}{\tau}=\frac{1}{2 \tau}\left[\left(\frac{p}{r}\right)^{\sigma}-\left(\frac{r}{p}\right)^{\sigma}\right] .
$$

is invertible via the inversion of the Radon transform on the Cormack's $\beta$-curves.

In particular for $\sigma=1 / m, m=1,2,3, \ldots$, there exists a closed form inverse formula

$\left.f(r, \theta)\right|_{\sigma=1 / m}=\frac{1}{2 \pi^{2}} \frac{d}{d r} \ln g(r) \int_{0}^{2 \pi} d \phi \int_{0}^{\infty} d \tau \frac{d}{d \tau}\left(\frac{\tau \widehat{f}(\tau, \phi)}{\sqrt{1+\tau^{2}}}\right) \frac{U_{m-1}(g(r) / \tau)}{T_{m}(g(r) / \tau)-\cos (\theta-\phi)}$,

where $T_{m}(x)$ and $U_{m}(x)$ are the Tschebycheff polynomials of first and second kind [23]

$$
T_{m}(\cos x)=\cos m x \quad \text { and } \quad U_{m-1}(\cos x)=\frac{\sin m x}{\sin x} .
$$

Proof: From eq. (17b) of [2], we retrieve the inversion formula for $F_{l}(t)$

$$
F_{l}(t)=\frac{1}{\pi t} \int_{0}^{t} d \tau \frac{\cosh \left(\frac{l}{\sigma} \cosh ^{-1}(t / \tau)\right)}{\sqrt{t^{2}-\tau^{2}}} \frac{d \widehat{F}_{l}(\tau)}{d \tau},
$$

Because eq. (26) does not include the consistency condition on the Radon data, the function under $\tau$-integration shows divergences, at large $l>2$, for $\tau \rightarrow 0$. A wellbehaved solution was derived later by Cormack in 1984 (see his eq. (15) in [22]). When transcribed to our $\sigma$-solution, it reads

$$
\begin{gathered}
F_{l}(g)=\frac{1}{\pi g} \int_{0}^{g} d \tau \widehat{F}_{l} l(\tau)\left\{\frac{\left(\frac{g}{\tau}-\sqrt{\frac{g^{2}}{\tau^{2}}-1}\right)^{l / \sigma}}{\sqrt{\frac{g^{2}}{\tau^{2}}-1}}+\sum_{k=[n / 2]+1}^{\infty} a_{k}\left(\frac{2 g}{\tau}\right)^{n+\delta-2 k}\right\} \\
-\frac{1}{\pi g} \int_{g}^{\infty} d \tau \widehat{F}^{\prime}{ }_{l}(\tau) \sum_{k=0}^{[n / 2]} a_{k}\left(\frac{2 g}{\tau}\right)^{n+\delta-2 k},
\end{gathered}
$$

whereby $n=0,1,2, \ldots, \quad l / \sigma=1+n+\delta, \quad 0<\delta<1$ and

$$
a_{k}=\frac{(-1)^{k}}{k !} \frac{\Gamma(1+n+\delta-k)}{\Gamma(1+n+\delta-2 k)} .
$$

Then using eq. (23), an inversion formula in terms of $\widehat{f}_{l}(\tau)$ can be obtained.

For $\sigma=1 / m$, with $m=1,2,3, \ldots$, Cormack was able to obtain a close form for the inversion formula (his eq. (21) in [22], in which $(t / q)$ is replaced by $(q / t)$ ). The transcription of his result to our variables yields precisely formula (25).

Remark 2.7 We may observe that the Radon transforms on curves given by eq. (7) cannot be inverted by circular harmonic decomposition. 


\section{A Radon transform on arcs of circles orthogonal to a fixed circle}

This section is devoted to the Radon transform on the curves given by eq. (22). For arbitrary $\sigma>0$, this transform has its novelty as a new class of Radon transform. Its inversion would go along the lines of the previous section. However in view of a possible application to Compton Scatter Tomography (CST), to be presented in section 4 , instead of describing the general inversion procedure for any $\sigma>0$, we choose to concentrate on the case $\sigma=1$ and give a self-contained presentation with relevant computational details.

\subsection{The family of circles $C_{p \tau}$}

The curve with $\sigma=1, C<0$ and the provisions c) of subsection 2.5, has the following equation in polar coordinates

$$
\cos \gamma=\frac{1}{2 \tau}\left(\frac{p}{r}+\frac{r}{p}\right), \quad \text { with } \quad \tau>0 \text { and } p>0 .
$$

It is clearly invariant under geometric inversion $(p / r) \leftrightarrow(r / p)$, and under the substitution $(r, \gamma) \rightarrow(-r, \gamma \pm \pi)$. Inspection shows that it is a circle $C_{p \tau}$ of radius $p \sqrt{\tau^{2}-1}$, with its center on a radial axis making an angle $\phi$ with $O x$, at a distance $p \tau$ from the origin and orthogonal to a fixed circle $\Gamma_{p}$ of radius $p$ and centered at the coordinate system origin $O$. The intersections points $S$ and $D$ of $C_{p \tau}$ with $\Gamma_{p}$ are also the tangency points to the full circle $C_{p \tau}$, of lines $O S$ and $O D$, see Fig. 2.

When eq. (28) is solved for $r$, one gets two solutions. With respect to the circle $\Gamma_{p}$, its outside (resp. inside) branch is given by $r=p\left(\tau \cos \gamma+\sqrt{\tau^{2} \cos ^{2} \gamma-1}\right)$ (resp. $\left.r=p\left(\tau \cos \gamma-\sqrt{\tau^{2} \cos ^{2} \gamma-1}\right)\right)$. The existence and reality of these solutions requires that $\left(-\pi / 2<-\gamma_{0}<\gamma<\gamma_{0}<\pi / 2\right)$, with $\gamma_{0}$ defined by $\tau^{2} \cos ^{2} \gamma_{0}=1$, which in turns implies that $\tau \geq 1$ (since $g(r)=(1 / 2)((p / r)+(r / p))>0$, its minimum is $\zeta=1$ at $r=p)$.

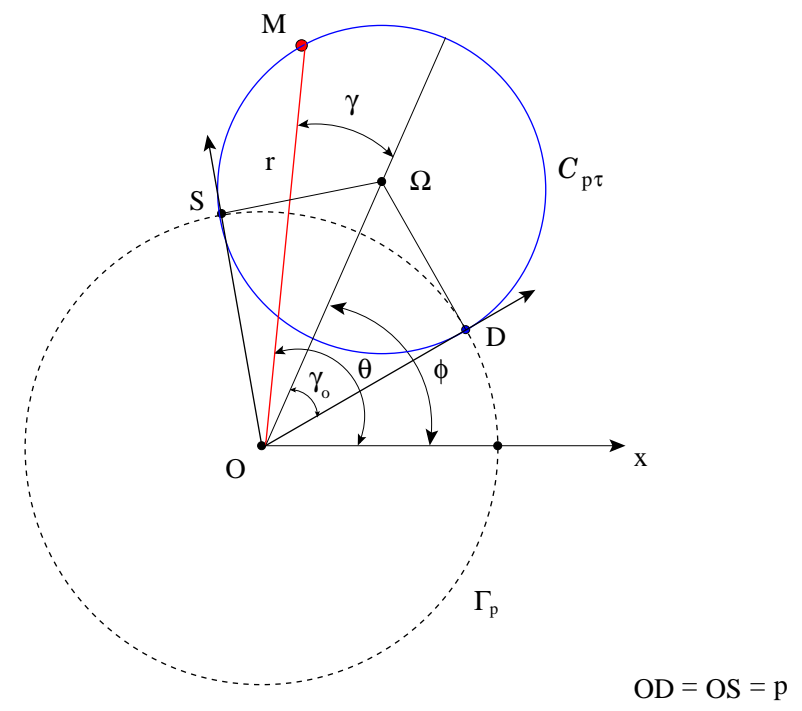

Figure 2. Circle $C_{p \tau}$ orthogonal to a fixed circle $\Gamma_{p}$ 


\subsection{Known cases of Radon transforms on circles in $\mathbb{R}^{2}$}

The problem of invertibility of Radon transforms on circles in $\mathbb{R}^{2}$ has been investigated in depth by many authors, see e.g. [10]. In particular, the invertibility of Radon transforms on circles of arbitrary radius but centered on restricted subsets $S$ of the plane has been established by Agranovsky and Quinto (see [24, 25]). This inversion problem is equivalent to the characterization of sets on which radial functions are dense in the sense of continuous functions in the plane. This is so except if $S$ is not contained in any set of the form of the union of rigidly displaced Coxeter system of $N$ lines and of a finite set $F$. The related problem of injectivity of Radon transform on circles has been also largely investigated in the literature [24, 25, 26], in particular for the Radon transform on circles with fixed radius [27].

The circles $C_{p \tau}$ have not appeared so far in the literature as support for Radon transforms in $\mathbb{R}^{2}$. The only known classes of circles $\oint$ on which a Radon transform can be defined and inverted with an explicit formula are:

- 1 - Circles going through the coordinate system origin. The Radon transform on such circles was found to be invertible first by A M Cormack in 1963 [1]. Interest on this case remained dormant for years until Cormack showed that they are a special case of his $\beta$-curves in [2]. Much later S J Norton used them to propose a modality for Compton Scatter Tomography (CST) [21].

- 2 - Circles of varying radius centered on a fixed circle. It was shown by S J Norton [28] how they arise in ultra-sound imaging with special circular geometry . This case has experienced now a revival of interest with thermo-acoustic imaging [6].

- 3 - Circles centered on a straight line. They have served in Radon transforms for modeling Synthetic Aperture Radar (SAR) imaging [4].

All these three classes of circles have been discussed thoroughly in the literature $[24,25,26]$.

- 4 - Recently while searching for a new modality for Compton Scatter Tomography, we have found that the class of arcs of circle subtended by a chord of fixed length and rotating around its center, can be the support of a new class of invertible Radon transforms [16]. A corresponding CST modality has been also suggested.

\subsection{The Radon transform on arcs of circle $C_{p \tau}^{+}$}

In this section we show that the Radon transform on circular arcs $C_{p \tau}^{+}$, which are orthogonal to the fixed circle $\Gamma_{p}$, but external to it, is invertible by Cormack's approach. It has the polar equation $r=p\left(\sqrt{\tau^{2} \cos ^{2} \gamma-1}+\tau \cos \gamma\right)$ and has a length $p\left(\pi+2 \gamma_{0}\right) \tan \gamma_{0}$, with $\gamma_{0}=\cos ^{-1}\left(\tau^{-1}\right)$.

Definition 3.1 The Radon transform $\widehat{f}(\tau, \phi)$ of functions $f(r, \theta) \in \mathcal{S}\left(\mathbb{R}^{2}\right)$ on $C_{p \tau}^{+}$is defined by the integral

$$
\widehat{f}(\tau, \phi)=\int_{-\gamma_{0}}^{\gamma_{0}} d \gamma \sqrt{\frac{\tau^{2}-1}{\tau^{2} \cos ^{2} \gamma-1}}(r f(r, \gamma+\phi))_{r=p}\left(\sqrt{\tau^{2} \cos ^{2} \gamma-1}+\tau \cos \gamma\right),
$$

$\S$ The general problem of Radon on arcs of curves has been considered in [20] 
Radon transforms on generalized Cormack's curves and a new Compton scatter tomography modality 12 or alternatively by

$$
\begin{gathered}
\hat{f}(\tau, \phi)= \\
\left.\int_{p}^{p\left(\tau+\sqrt{\tau^{2}-1}\right)} d r \sqrt{\frac{\tau^{2}-1}{\tau^{2}-\frac{1}{4}\left(\frac{p}{r}+\frac{r}{p}\right)^{2}}}(f(r, \gamma+\phi)+f(r,-\gamma+\phi))\right|_{\gamma=\cos ^{-1} \frac{1}{2 \tau}\left(\frac{p}{r}+\frac{r}{p}\right)} .
\end{gathered}
$$

In eq. (29), the factor $\left(\sqrt{\tau^{2} \cos ^{2} \gamma-1}\right)^{-1}$ introduces an integrable singularity near the integration limits $\pm \gamma_{0}$ and the $\gamma$-integration remains well-defined. We note that $\widehat{f}(1, \phi)=0$ and that $\widehat{f}(\tau, \phi) \in \mathcal{S}\left(\mathbb{R}^{2}\right)$.

Alternatively $\widehat{f}(\tau, \phi)$, as a function of $(\tau, \phi) \in[1, \infty[\times[0,2 \pi]$ can be reformulated as an integral transform with a $r$-delta function kernel $\mathcal{K}_{C_{p \tau}^{+}}(\tau, \phi \mid r, \theta)$ as

$$
\widehat{f}(\tau, \phi)=\int_{-\gamma_{0}}^{\gamma_{0}} d \gamma \int_{\mathbb{R}} d r r f(r, \gamma+\phi) \mathcal{K}_{C_{p \tau}^{+}}(\tau, \phi \mid r, \theta)
$$

where the kernel is

$\mathcal{K}_{C_{p \tau}^{+}}(\tau, \phi \mid r, \theta)=\sqrt{\frac{\tau^{2}-1}{\tau^{2} \cos ^{2}(\theta-\phi)-1}} \delta\left(r-p\left(\sqrt{\tau^{2} \cos ^{2}(\theta-\phi)-1}+\tau \cos (\theta-\phi)\right)\right)$

Thus for given $(\tau, \phi)$, the kernel support in $(r, \theta)$-space is $C_{p \tau}^{+} \|$.

\subsection{Adjoint transform}

Definition 3.2 The adjoint Radon transform of a real-valued, smooth and rapidly decreasing function $\widehat{h}(\tau, \phi)$ is $h(r, \theta)$, given by

$$
h(r, \theta)=\int_{0}^{2 \pi} d \phi \int_{1}^{\infty} d \tau \mathcal{K}_{C_{p \tau}^{+}}^{+}(r, \theta \mid \tau, \phi) \widehat{h}(\tau, \phi)
$$

To find $\mathcal{K}_{C_{p \tau}^{+}}^{+}(r, \theta \mid \tau, \phi)$ we rewrite the kernel in eq. (33) in terms of the $\tau$-variable in the delta-function

$$
\begin{aligned}
& \delta\left(r-p\left(\sqrt{\tau^{2} \cos ^{2}(\theta-\phi)-1}+\tau \cos (\theta-\phi)\right)\right)= \\
& \delta\left(\tau-\frac{\frac{1}{2}\left(\frac{p}{r}+\frac{r}{p}\right)}{\cos (\theta-\phi)}\right) \frac{\sqrt{\tau^{2} \cos ^{2}(\theta-\phi)-1}}{r \cos (\theta-\phi)} .
\end{aligned}
$$

Let

$$
g=\frac{1}{2}\left(\frac{p}{r}+\frac{r}{p}\right)
$$

\| We can verify that the normalization is correct since the integral on the function 1 with this delta function yields the length of the circumference. 
Inspection show that $g$ as a function of $r$, is always $g>1$ and $g$ attains its minimum 1 at $r=p$. Thus for a given point $(r, \theta)$, the kernel of the adjoint transform is

$$
\mathcal{K}_{C_{p \tau}^{+}}^{+}(r, \theta \mid \tau, \phi)=\frac{\sqrt{\tau^{2}-1}}{r} \delta(\tau \cos (\phi-\theta)-g) .
$$

Its support in $(\tau, \phi)$-space (or Radon space) is just the curve of equation

$$
\tau=\frac{g}{\cos (\phi-\theta)}
$$

This is precisely the equation of a straight line in polar coordinates $(\tau, \phi)$, located at a distance $g$ from the coordinate origin. Since $g>1$, the set of these straight lines in the $(\tau, \phi)$ plane lies outside the unit circle. Thus the adjoint Radon transform of the function $\widehat{h}(\tau, \phi)$ is the usual Radon transform on straight lines (external to the unit circle) in $(\tau, \phi)$-space but for the function $\sqrt{\tau^{2}-1} \widehat{h}(\tau, \phi)$ with the kernel $\delta(g-\tau \cos (\phi-\theta))$, which is $p\left(\sqrt{g^{2}-1}+g\right) h\left(p\left(\sqrt{g^{2}-1}+g\right), \theta\right)=r h(r, \theta)$.

\subsection{The integral transform for circular harmonic components}

Under the above assumptions, Proposition 2.3 takes the form:

Proposition 3.3 Let $f_{l}(r)$ (resp. $\widehat{f}_{l}(\tau)$ ) be the circular component of $f(r, \theta)$ (resp. of $\widehat{f}(\tau, \phi)$ ) and define $\widehat{F}_{l}(\tau)$ (resp. $\left.F_{l}(g)\right)$ by

$$
\widehat{F}_{l}(\tau)=\frac{\widehat{f_{l}}(\tau)}{\sqrt{\tau^{2}-1}}, \quad \text { and } \quad F_{l}(g)=\frac{p\left(g+\sqrt{g^{2}-1}\right) f_{l}\left(p\left(g+\sqrt{g^{2}-1}\right)\right)}{\sqrt{g^{2}-1}} .
$$

Then $\widehat{F}_{l}(\tau)$ the Tschebycheff transform of the first kind of $F_{l}(g)$

$$
\widehat{F}_{l}(\tau)=2 \int_{1}^{\tau} d g \frac{T_{l}(g / \tau)}{\sqrt{1-\left(\frac{g}{\tau}\right)^{2}}} F_{l}(g)
$$

where $T_{l}(x)$ is the Tschebycheff polynomial of the first kind [23].

Eq. (39) is well-defined since the singularities of the integrand at $g=(1, \tau)$ are both integrable. It differs only from the equation for the circular harmonic components of the Radon problem on circles through the origin of A M Cormack by the value of the lower integration bound (see eq. (22) in [1]). Thus most of properties proved for the Cormack's problem remain valid here [2].

Proof: In eq. (29), we insert the circular harmonic decomposition of $f(r, \theta)$. Then exchanging the discrete $l$-summation with the $\gamma$-integration we can identify the circular harmonic component $\widehat{f}_{l}(\tau)$ of $\widehat{f}(\tau, \phi)$ as

$$
\widehat{f}_{l}(\tau)=2 \int_{-\gamma_{0}}^{\gamma_{0}} d \gamma \sqrt{\frac{\tau^{2}-1}{\tau^{2} \cos ^{2} \gamma-1}}\left(r f_{l}(r)\right)_{r=p\left(\tau \cos \gamma+\sqrt{\tau^{2} \cos ^{2} \gamma-1}\right)} e^{i l \gamma} .
$$

This result can be rewritten as an integration on $\left[0, \gamma_{0}\right]$, in which $e^{i l \gamma}$ replaced by $2 \cos l \gamma$. Then changing the $\gamma$-integration into $r$-integration, eq. (40) becomes

$$
\widehat{f}_{l}(\tau)=2 \int_{p\left(\tau+\sqrt{\tau^{2}+1}\right)}^{p} d r \sqrt{\frac{\tau^{2}-1}{1-\frac{\left(\frac{p}{r}+\frac{r}{p}\right)^{2}}{4 \tau^{2}}}} \frac{\frac{1}{2 \tau}\left(\frac{p}{r}-\frac{r}{p}\right)}{\sqrt{\tau^{2} \cos ^{2} \gamma-1}} \cos l\left(\frac{1}{2 \tau}\left(\frac{p}{r}+\frac{r}{p}\right)\right) f_{l}(r) .
$$


Radon transforms on generalized Cormack's curves and a new Compton scatter tomography modality14

Now using eq. (28), we see that

$$
\frac{\frac{1}{2 \tau}\left(\frac{p}{r}-\frac{r}{p}\right)}{\sqrt{\tau^{2} \cos ^{2} \gamma-1}}=\operatorname{sgn} \frac{1}{2 \tau}\left(\frac{p}{r}-\frac{r}{p}\right)=-1, \text { for } r>p .
$$

Thus with $g$ given by eq. (36), eq. (41) becomes

$$
\frac{\widehat{f_{l}}(\tau)}{\sqrt{\tau^{2}-1}}=2 \int_{p}^{p\left(\tau+\sqrt{\tau^{2}-1}\right)} d r \frac{\cos l \cos ^{-1}(g / \tau)}{\sqrt{1-\frac{g^{2}}{\tau^{2}}}} f_{l}(r) .
$$

The last step consists in going over the variable $g$ as integration variable with

$$
\frac{d r}{d g}=\frac{1}{-\frac{1}{2 r}\left(\frac{p}{r}-\frac{r}{p}\right)}=p \frac{g+\sqrt{g^{2}-1}}{\sqrt{g^{2}-1}}, \text { and } F_{l}(g)=\left(\frac{r f_{l}(r)}{-\frac{1}{2}\left(\frac{p}{r}-\frac{r}{p}\right)}\right)_{r=p\left(g+\sqrt{g^{2}-1}\right)} .
$$

With $T_{l}(x)$ as defined in Theorem 2.6, eq. (39) is established.

Eq. (39) has precisely the form of the equation for circular components of the Radon on circles though the origin [2], except that the lower integration bound is not 0 but 1 (recall that $\tau \geqslant 1$ ).

\subsection{Consistency conditions}

Proposition 3.4 For $l \in \mathbb{N}_{+}$and $k=l, l-2, l-4, \ldots>0$, the following integral vanishes:

$$
\int_{1}^{\infty} d \tau \tau^{-k} \frac{\widehat{f}_{l}(\tau)}{\sqrt{\tau^{2}-1}}=0
$$

Proof: Using proposition 3.3, we have with $k>0$

$$
\begin{gathered}
\int_{1}^{\infty} d \tau \tau^{-k} \frac{\widehat{f}_{l}(\tau)}{\sqrt{\tau^{2}-1}}= \\
2 \int_{1}^{\infty} \frac{d \tau}{\tau^{k}} \int_{1}^{\tau} d g \frac{\cos l\left(\cos ^{-1}\left(\frac{g}{\tau}\right)\right)}{\sqrt{1-\left(\frac{g}{\tau}\right)^{2}}} \frac{p\left(g+\sqrt{g^{2}-1}\right) f_{l}\left(p\left(g+\sqrt{g^{2}-1}\right)\right)}{\sqrt{g^{2}-1}} .
\end{gathered}
$$

Exchanging integration order on the right-hand-side of eq. (45) as in [2], we have

$$
\int_{1}^{\infty} d g \frac{p\left(g+\sqrt{g^{2}-1}\right) f_{l}\left(p\left(g+\sqrt{g^{2}-1}\right)\right)}{\sqrt{g^{2}-1}} \int_{g}^{\infty} d \tau \tau^{-k} \frac{\cos l\left(\cos ^{-1}\left(\frac{g}{\tau}\right)\right)}{\sqrt{1-\left(\frac{g}{\tau}\right)^{2}}} .
$$

In the $\tau$-integral we change to the variable $\nu$ (for fixed $g>1$ ), defined by $\tau=g / \cos \nu$. Then

$$
\int_{g}^{\infty} d \tau \tau^{-k} \frac{\cos l\left(\cos ^{-1}\left(\frac{g}{\tau}\right)\right)}{\sqrt{1-\left(\frac{g}{\tau}\right)^{2}}}=g^{-k+1} \int_{0}^{\pi / 2} d \nu \cos l \nu \cos ^{k-2} \nu
$$


Radon transforms on generalized Cormack's curves and a new Compton scatter tomography modality 15

The value of the $\nu$-integral is given in [23] as

$$
\int_{0}^{\pi / 2} d \nu \cos l \nu \cos ^{k-2} \nu=\frac{\pi}{(k-1) 2^{k-1}} \frac{\Gamma(k)}{\Gamma\left(\frac{k+l}{2}\right) \Gamma\left(\frac{k-l}{2}\right)} .
$$

For $l>0$ this integral vanishes for $k=l, l-2, l-4, \ldots>0$. For $l<0$ we take the poles of the other gamma-function factor. $\square$

This consistency condition shall be used to deduce a closed form of the reconstruction formula, as in [22]. We have not investigated at this point the connection to special orthogonal polynomials, as done in $[3,29]$ for the usual Radon transform, but we have the following corollary:

Corollary 3.5 For $t<\tau$,

$$
\int_{1}^{\infty} d \tau U_{l-1}(t / \tau) \frac{\widehat{f}_{l}(\tau)}{\tau \sqrt{\tau^{2}-1}}=0
$$

where $U_{l-1}(\cos \nu)=\sin l \nu / \sin \nu$ is the Tchebyscheff polynomial of second kind.

Proof: Using the explicit expression of $U_{l-1}(t / \tau)$, as given in [23], we have

$$
\frac{t}{\tau} U_{l-1}(t / \tau)=\sum_{m=0}^{(l-1) / 2} \sum_{n=0}^{m}\left(\begin{array}{c}
l \\
2 m+1
\end{array}\right)\left(\begin{array}{c}
m \\
n
\end{array}\right)(-1)^{n-m}\left(\frac{t}{\tau}\right)^{l-2(m+1-n)}
$$

where $l-2(m+1-n)>0$. Thus eq. (47) is a linear sums of terms of the type of eq. $(44)$.

3.7. Inversion formula for the circular harmonic components

Theorem 3.6 Under the above assumptions, the function $f(r, \theta)$ can be reconstructed via its circular harmonic component $f_{l}(r)$, given by

$$
f_{l}(r)=\frac{1}{2 \pi r}\left(\frac{r}{p}-\frac{p}{r}\right)\left\{\frac{d}{d t} \int_{1}^{t} d \tau \frac{\cosh \left(l \cosh ^{-1}\left(\frac{t}{\tau}\right)\right)}{\sqrt{\left(t^{2}-\tau^{2}\right)\left(\tau^{2}-1\right)}} \widehat{f}_{l}(\tau)\right\}_{t=\frac{1}{2}\left(\frac{p}{r}+\frac{r}{p}\right)}
$$

for $l \in \mathbb{Z}$.

Proof: We multiply both sides of eq. (39) by

$$
\int_{1}^{t} d \tau \frac{\cosh \left(l \cosh ^{-1}\left(\frac{t}{\tau}\right)\right)}{\tau \sqrt{\left(\frac{t}{\tau}\right)^{2}-1}}
$$

Since the integrand on the right-hand-side of eq. (39) is smooth except for integrable singularities in the plane $(\tau, g)$, we can interchange the integration order and rearrange the integrations as follows

$$
\int_{1}^{t} d \tau \frac{\cosh \left(l \cosh ^{-1}\left(\frac{t}{\tau}\right)\right)}{\tau \sqrt{\left(\frac{t}{\tau}\right)^{2}-1}} \widehat{F}_{l}(\tau)=2 \int_{1}^{t} d g F_{l}(g) \int_{g}^{t} d \tau \frac{\cosh \left(l \cosh ^{-1}\left(\frac{t}{\tau}\right)\right)}{\tau \sqrt{\left(\frac{t}{\tau}\right)^{2}-1}} \frac{\cos l\left(\cos ^{-1}\left(\frac{g}{\tau}\right)\right)}{\sqrt{1-\left(\frac{g}{\tau}\right)^{2}}}
$$


Radon transforms on generalized Cormack's curves and a new Compton scatter tomography modality 16

The $\tau$-integral on the right-hand-side of eq. (50) has the value $\pi / 2$, as shown in $[2,17]$. Consequently

$$
\int_{1}^{t} d \tau \frac{\cosh \left(l \cosh ^{-1}\left(\frac{t}{\tau}\right)\right)}{\tau \sqrt{\left(\frac{t}{\tau}\right)^{2}-1}} \widehat{F}_{l}(\tau)=\pi \int_{1}^{t} d g F_{l}(g) .
$$

$F_{l}(t)$ is recovered by deriving with respect to $t$ on both sides of this equation, i.e.

$$
F_{l}(t)=\frac{1}{\pi} \frac{d}{d t} \int_{1}^{t} d \tau \frac{\cosh \left(l \cosh ^{-1}\left(\frac{t}{\tau}\right)\right)}{\tau \sqrt{\left(\frac{t}{\tau}\right)^{2}-1}} \widehat{F}_{l}(\tau)
$$

Then (49) is obtained after switching back to the original $\widehat{f}(\tau, \phi)$ and $f(r, \theta)$, via eq. (38), in which $g$ plays the role of $t$ here.

As observed by A M Cormack in [2], this result demonstrates the "hole" theorem for the Radon transform on the arcs of circle $C_{p \tau}^{+}$: it is only necessary to know $\widehat{f}_{l}(\tau)$ for

$$
1<\tau<\frac{1}{2}\left(\frac{p}{r}+\frac{r}{p}\right)
$$

in order to determine $f(r, \theta)$.

\subsection{A closed form of the inversion formula}

Following [22], a closed form the inversion formula can be established.

Proposition 3.7 Taking into account the consistency conditions (44), the final closed form of the reconstruction formula is

$$
\begin{gathered}
f(r, \theta)=\frac{1}{2 \pi r}\left(\frac{r}{p}-\frac{p}{r}\right) \times \\
P . V . \int_{0}^{2 \pi} \frac{d \phi}{2 \pi} \frac{1}{\cos (\theta-\phi)}\left\{P . V . \int_{1}^{\infty} d \tau\left(\frac{d}{d \tau} \frac{\widehat{f}(\tau, \phi)}{\sqrt{\left(\tau^{2}-1\right)}}\right) \frac{1}{\frac{1}{2}\left(\frac{p}{r}+\frac{r}{p}\right)-\tau \cos (\theta-\phi)}\right\} \cdot(52
\end{gathered}
$$

where P.V. denotes the principal value of the integral.

Proof: We rewrite eq. (49) in terms of the Tchebyscheff polynomial $T_{l}(t / \tau)$ for $1<\tau<t$

$$
f_{l}(r)=\frac{1}{2 \pi r}\left(\frac{r}{p}-\frac{p}{r}\right)\left\{\frac{d}{d t} \int_{1}^{t} d \tau \frac{T_{|l|}\left(\frac{t}{\tau}\right)}{\sqrt{\left(t^{2}-\tau^{2}\right)\left(\tau^{2}-1\right)}} \widehat{f}_{l}(\tau)\right\}_{t=\frac{1}{2}\left(\frac{p}{r}+\frac{r}{p}\right)}
$$

and use the identity (16) in [22], to express the $\tau$-integral in eq. (53) as a sum of two integrals, i.e.

$$
\int_{1}^{t} \frac{d \tau}{\tau} \frac{\left(\frac{t}{\tau}-\sqrt{\left(\frac{t}{\tau}\right)^{2}-1}\right)^{|l|}}{\sqrt{\left(\frac{t}{\tau}\right)^{2}-1}} \frac{\widehat{f}_{l}(\tau)}{\sqrt{\tau^{2}-1}}+\int_{1}^{t} \frac{d \tau}{\tau} U_{|l|-1}\left(\frac{t}{\tau}\right) \frac{\widehat{f}_{l}(\tau)}{\sqrt{\tau^{2}-1}} .
$$

Then eq. (47) allows to rewrite the second integral in the previous equation as

$$
\int_{1}^{t} \frac{d \tau}{\tau} U_{|l|-1}\left(\frac{t}{\tau}\right) \frac{\widehat{f}_{l}(\tau)}{\sqrt{\tau^{2}-1}}=-\int_{t}^{\infty} \frac{d \tau}{\tau} U_{|l|-1}\left(\frac{t}{\tau}\right) \frac{\widehat{f}_{l}(\tau)}{\sqrt{\tau^{2}-1}}
$$


Radon transforms on generalized Cormack's curves and a new Compton scatter tomography modality 17 which is well-defined since $1<t<\tau$.

Next we need the sums of two series, which are given by the following lemmas.

Lemma 3.8 For $1<\tau<t$, one has

$$
1+2 \sum_{l=1}^{\infty}\left(\frac{t}{\tau}-\sqrt{\left(\frac{t}{\tau}\right)^{2}-1}\right)^{l} \cos l(\theta-\phi)=\frac{-\sqrt{\left(\frac{t}{\tau}\right)^{2}-1}}{\cos (\theta-\phi)-\frac{t}{\tau}} .
$$

Proof: Since

$$
0<\left(\frac{t}{\tau}-\sqrt{\left(\frac{t}{\tau}\right)^{2}-1}\right)<1
$$

application of the summation formula on page 468 of [23] yields the quoted result.

Lemma 3.9 For $0<t / \tau<1$, we have

$$
\begin{aligned}
\sum_{l \in \mathbb{Z}} U_{|l|-1}\left(\frac{t}{\tau}\right) e^{i l(\theta-\phi)} & =\lim _{a \rightarrow 0_{+}} \frac{1}{\sin \cos ^{-1}(t / \tau)} \sum_{l \in \mathbb{Z}} \sin \left(|l| \cos ^{-1}(t / \tau)\right)\left(e^{-l a+i l(\theta-\phi)}\right) \\
& =\frac{1}{\cos (\theta-\phi)-\frac{t}{\tau}} .
\end{aligned}
$$

Proof: For given $a>0$, using $U_{|l|-1}\left(\frac{t}{\tau}\right)=\sin \left(|l| \cos ^{-1}(t / \tau)\right) / \sin \cos ^{-1}(t / \tau)$, and $U_{-1}(x)=0$, the $l$-sum may be split into a sum of two terms: one with $l=1,2, \ldots \infty$ and the other with $l=-1,-2, \ldots-\infty$. By relabeling the negative $l$ index, we obtain

$$
\begin{gathered}
\sum_{l \in \mathbb{Z}} U_{|l|-1}\left(\frac{t}{\tau}\right) e^{i l(\theta-\phi)}=\lim _{a \rightarrow 0_{+}} \\
\frac{1}{\sin \cos ^{-1}(t / \tau)} \sum_{l=1}^{\infty}\left(\sin l \cos ^{-1}(t / \tau)\left(e^{-a+i(\theta-\phi)}\right)^{l}+\sin l \cos ^{-1}(t / \tau)\left(e^{-a-i(\theta-\phi)}\right)^{l}\right) .
\end{gathered}
$$

The two sums on the right-hand-side can be evaluated using a formula on page 468 of [23] for given $a>0$. Then after taking the limit $a \rightarrow 0$, one gets

$$
\sum_{l \in \mathbb{Z}} U_{|l|-1}\left(\frac{t}{\tau}\right) e^{i l(\theta-\phi)}=\frac{1}{\cos (\theta-\phi)-(t / \tau)}
$$

which is the expected result.

Next after writing $\widehat{f}_{l}(\tau)$ as the angular Fourier component of $\widehat{f}(\tau, \phi)$, (see eq. (4)) in the expression of $f_{l}(r)$ given by eq. (53), we compute $f(r, \theta)$ as $\sum_{l \in \mathbb{Z}} f_{l}(r) \exp i l \theta$. The reconstruction formula appears after applying the results of the two previous lemmas as

$f(r, \theta)=\frac{1}{2 \pi r}\left(\frac{r}{p}-\frac{p}{r}\right) \int_{0}^{2 \pi} \frac{d \phi}{2 \pi}\left\{\frac{d}{d t}\left[\mathrm{P} . \mathrm{V} . \int_{1}^{\infty} d \tau \frac{\widehat{f}(\tau, \phi)}{\sqrt{\left(\tau^{2}-1\right)}} \frac{1}{t-\tau \cos (\theta-\phi)}\right] \int_{t=\frac{1}{2}\left(\frac{p}{r}+\frac{r}{p}\right)}\right.$. 
As $t$ does no longer appear as integration bound, the $t$-derivative can be shoved under the $\tau$-integration. It generates a factor $(t-\tau \cos (\theta-\phi))^{-2}$ in the integrand, with an apparent bad divergence. However as shown in [30], a regularization procedure can be applied so that the integral transform with kernel $(t-\tau \cos (\theta-\phi))^{-1}$ acts on the $\tau$-derivative of $\widehat{f}(\tau, \phi) / \sqrt{\left(\tau^{2}-1\right)}$. This is formula (52). Alternatively one may also use partial integration on $\tau$ with the boundary values $\left(\widehat{f}(\tau, \phi) / \sqrt{\tau^{2}-1}\right)_{\tau=1, \infty}=0$, (due to eq. (39) and its own structure), to obtain the final result (52).

\subsection{The Radon transform on arcs of circle $C_{p \tau}^{-}$}

If the inner arc of circle is used to define the Radon transform of a function $f(r, \theta)$, one should take the the equation $r=p\left(\tau \cos \gamma-\sqrt{\tau^{2} \cos ^{2} \gamma-1}\right)$. There are only minor differences in the calculations as compared to the previous case and one ends up exactly with the same equation for the circular harmonic components of proposition 3.3. Thus the previous inversion procedure will go through.

\section{A suggested modality for Compton Scatter Tomography}

\subsection{On Compton scatter tomography}
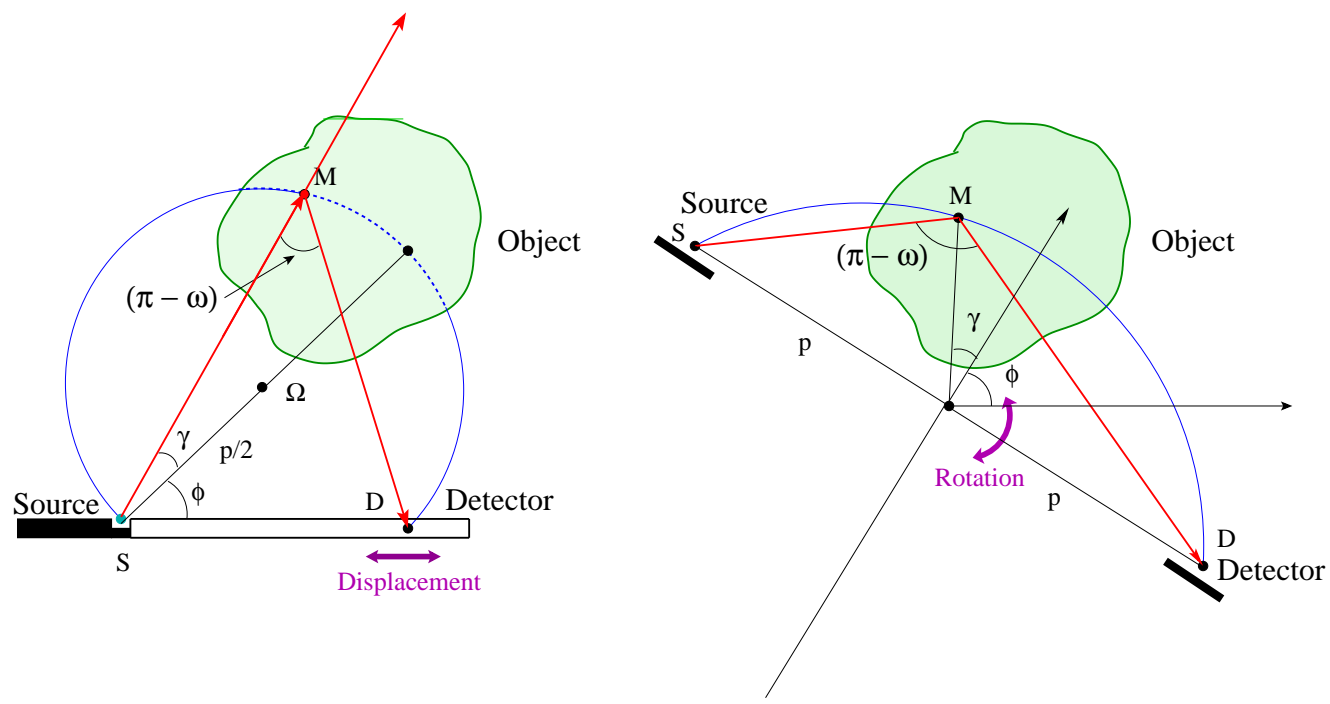

Figure 3. Existing Compton Scatter Tomography modalities associated to two classes of circular arcs

To image the hidden parts of objects of interest, transmission- or emission tomography is nowadays widely used. The flux density of ionizing radiation (i.e. $\mathrm{X}$ - or gamma rays) with definite energy $E_{0}$ is measured at the end of linear paths along all possible space orientations. In transmission tomography $\Phi$, the data is the cumulated attenuation of the radiation pencil with energy $E_{0}$, from an emission external source 
to a detection site. In emission tomography ${ }^{+}$, the detector records the integral of the object emitted radio-activity density along a given straight line path, which, in a first approximation, has not been attenuated by absorption or by Compton scattering. In both cases, the acquired data is represented by the usual Radon transform of a physical density function characterizing the object (linear attenuation coefficient or radio-activity emission density).

Compton Scatter Tomography (CST) is an alternative imaging technique which relies on the detection of Compton scattered radiation produced by an object when it is exposed to a external radiation source. The relevant quantity describing the object is now the object electronic density. This imaging principle was proposed first by P G Lale [31] and has evolved into many variants. The pioneering Compton scatter imaging methods are classified according to the way measurement of the spatial distribution of scatter radiation is done or the number of simultaneous volume elements being scanned: i.e. point by point, line by line, or plane by plane (for a complete account see the review [32]).

In 1978 instead of performing local step-by-step measurements, N N Kondic pointed out that scattered radiation data can be acquired as an integral of the electronic density along isogonic arcs of circle of constant scattered energy [33]. The idea was later on expanded in [34] by T H Prettyman, who realized that the data should be collected at all spatial orientations of the pair source-detector (or equivalently of the object). But having no analytic reconstruction formula adapted to this modality of data acquisition, he used standard numerical methods to reconstruct the object electron density.

In 1994, S J Norton showed that Kondic's idea can be implemented with a fixed point-like radiation source $\mathbf{S}$ and a point-like detector $\mathbf{D}$ moving on a straight line passing through $\mathbf{S}$ [21]. In this way, the collected data by the detector is essentially the Radon transform of the electronic density on circles of variable radius and going through S. Since such Radon transforms admit readily an analytic inverse [2], a natural reconstruction algorithm can be set up.

Recently, the inversion of a Radon transform on circular arcs subtended by a chord of fixed length but rotating about its center, has been established [16]. Its explicit inversion formula is precisely suited for the CST imaging procedure proposed by Prettyman in 1991.

Thus it is natural to ask whether the Radon transform on circles orthogonal to a fixed circle of section 3 may be of use for some new type of CST modality.

\subsection{A new Compton Scatter Tomography modality?}

In this section, we propose to give an affirmative answer to this question. This is illustrated by figure 4 .

A calibrated point source $\mathbf{S}$ of $\gamma$-rays, located on a circle $\Gamma_{p}$, emits photons of energy $E_{0}$. A lead shield nearby confines this emitted radiation to a half-space. An object, placed out side the circle $\Gamma_{p}$, is irradiated by the incoming radiation. Compton scattered radiation by the object electric charges is selectively registered, at different scattering energies $E$, by a point detector $\mathbf{D}$, also located on the circle $\Gamma_{p}$.

A scattered photon of energy $E<E_{0}$ is deviated by an angle $\omega$ from his initial propagation direction, after a Compton collision with an electron. $\omega$ is given by the

+ i.e. Single Photon Emission Computed Tomography (SPECT) or Positron Emission Tomography (PET). 


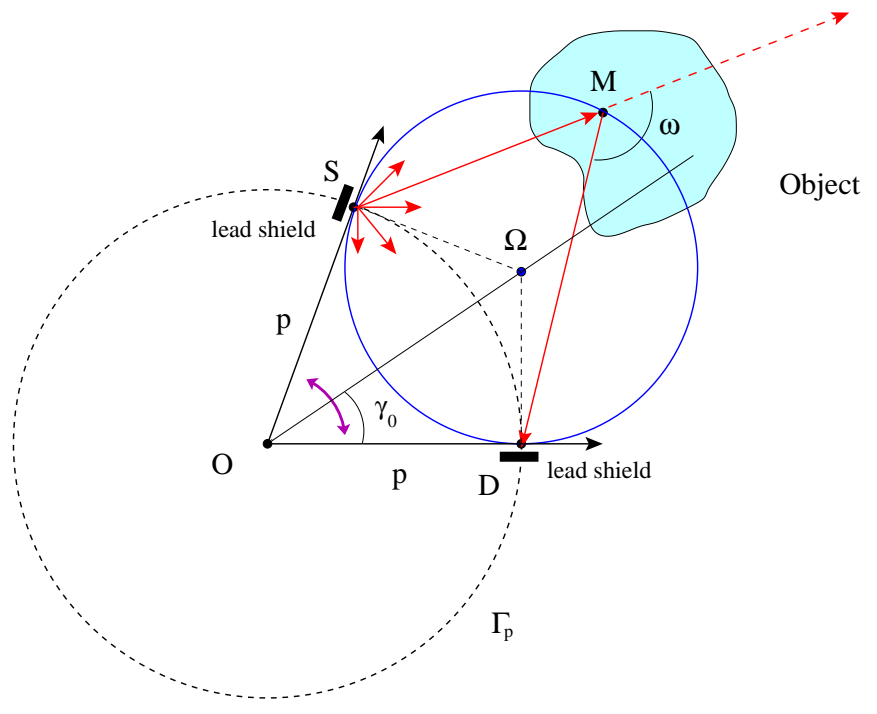

Figure 4. New Compton Scatter Tomography modality associated with external circular $\operatorname{arcs} C_{p \tau}^{+}$

so-called Compton relation $E=E_{0} /(1+\epsilon(1-\cos \omega))$, where $\epsilon=E_{0} / m c^{2}, m$ the electron rest mass and $c$ the light velocity in vacuum. From fig. 4, we can see that $\omega=\gamma_{0}+\pi / 2$ : this would mean that the measured data is due to Compton backscattered radiation, i.e. $(\pi / 2<\omega<\pi)$. We recall that $\cos \gamma_{0}=1 / \tau$. Thus when only photons of energy $E$ are registered, the photon flux density reaching $\mathbf{D}$ is proportional to the integral of the electron density $f(r, \theta)$ on $C_{p \tau}^{+}$. Radon data for reconstruction can be generated by rotating rigidly the pair of points $\mathbf{S}$ and $\mathbf{D}$ around the center $\mathbf{O}$ for all allowable relative angular separation $2 \gamma_{0}<\pi$. In this schematic presentation, in which only the essential idea is stressed, Compton kinematic factor, attenuation and photometric dispersion of photon propagation have not been taken into account in the reconstruction of $f(r, \theta)$ by formula (52). These points will be discussed in the next subsection.

\subsection{Inclusion of Compton kinematic factor, attenuation and photometric effects}

In general the Compton kinematic factor, which includes the Compton differential cross section, is solely a function of $\omega$, or of $\tau$ via $\tan \gamma_{0}=\sqrt{\tau^{2}-1}$, therefore its inclusion as a factor $P(\tau)$, would not alter the invertibility of the Radon transform. Radiation flux density emitted by a point source is weakened, after traveling a distance $r$ by a factor $1 / r^{n}$ with $n=1,2$ in two- and three-dimensional space. Accounting for this effect means inserting a factor $(S M \times M D)^{-n}$ in the definition of the Radon transform (29). Fortunately this product can be exactly evaluated and reexpressed as a product of a function of $r$ and a function of $\tau$. The distances $S M$ and $M D$ in the triangles $O S M$ and $O M D$ of fig. 4 are given by

$$
S M^{2}=p^{2}+r^{2}-2 p r \cos \left(\gamma_{0}-\gamma\right) \text { and } M D^{2}=p^{2}+r^{2}-2 p r \cos \left(\gamma_{0}+\gamma\right) .
$$

Using $\cos \gamma_{0}=\tau^{-1}$ and eq. (28), we can put their product under the form

$$
S M^{2} M D^{2}=\left(1-\frac{1}{\tau^{2}}\right)\left(r^{2}-p^{2}\right)^{2}=\sin ^{2} \gamma_{0}\left(r^{2}-p^{2}\right)^{2} .
$$


Eq. (29) now reads

$\widehat{f}(\tau, \phi)=P(\tau) \int_{-\gamma_{0}}^{\gamma_{0}} d \gamma \sqrt{\frac{\tau^{2}-1}{\tau^{2} \cos ^{2} \gamma-1}}\left[\frac{r f(r, \gamma+\phi)}{\left(1-\frac{1}{\tau^{2}}\right)^{n / 2}\left(p^{2}-r^{2}\right)^{n}}\right]_{r=p\left(\sqrt{\tau^{2} \cos ^{2} \gamma-1}+\tau \cos \gamma\right)}$.

A quick check shows that the invertibility of the transform via circular harmonic components remains valid. However the inclusion of a constant linear attenuation coefficient will not work in this scheme as already noticed in the case of [16].

\subsection{Advantages in applications}

The present modality, based on arcs of circle $C_{p \tau}^{+}$, is well adapted for imaging objects outside and far away from the circle $\Gamma_{p}$. The scanning $\phi$-rotation may be then conveniently replaced by a back-and-forth sweep motion (radar antenna type of motion) inside an angular sector. It generates a two-dimensional image of the hidden part of an object slice. A true three-dimensional image can be obtained next by axially superposing these two-dimensional images.

This is particularly interesting when the object is far away from the apparatus. Such a situation may arise in many situations: medical imaging, non-destructive industrial testing, geological prospection, etc. One important instance of application is the image reconstruction of objects situated on one side of a semi-infinite medium such as objects immersed below the sea level or objects buried under the earth surface. In particular, this modality may provide an interesting way for detection and imaging land (or sea) mines in a territory defense context and therefore it positions itself as a competitor to some other gamma-ray imaging processes advocated in recent years, e.g. see [35].

Now for objects of small sizes, one may use the modality based on arcs of circle $C_{p \tau}^{-}$. The object may placed within the circle $\Gamma_{p}$. The rotational motion of this pair for each opening angle $\gamma_{0}$ is easily realized in this case, as shown in fig. 5 . The pair source-detector has now a different lead shield arrangement, which in fact can be a circular belt radiation shielding around $\Gamma_{p}$ and is probably the most adequate protection against stray radiation during operation. Finally $p$ remains an adjustable parameter which may be used to fit the size of scanned objects.

\section{Conclusion and perspectives}

In this paper we have described a way of finding curves in $\mathbb{R}^{2}$ on which Radon transforms can be defined and inverted analytically through circular harmonic component decomposition. Such curves are called generalized Cormack's curves. They are solutions of a non-linear first-order differential equation and fall into three groups corresponding to the value and the sign of a free parameter $C$. Two groups of solutions are known from previous works $[2,16]$. The third one is new and its simplest representative is the class of circles orthogonal to a fixed circle centered at the origin. We have explicitly obtained the analytic inversion formula for this last case. This result suggests a new way for doing Compton Scatter Tomography, which might well be adapted for large objects hidden below the surface of a large medium as well as for smaller objects in a finite geometry. Thus such modality could find applications in numerous contexts. 


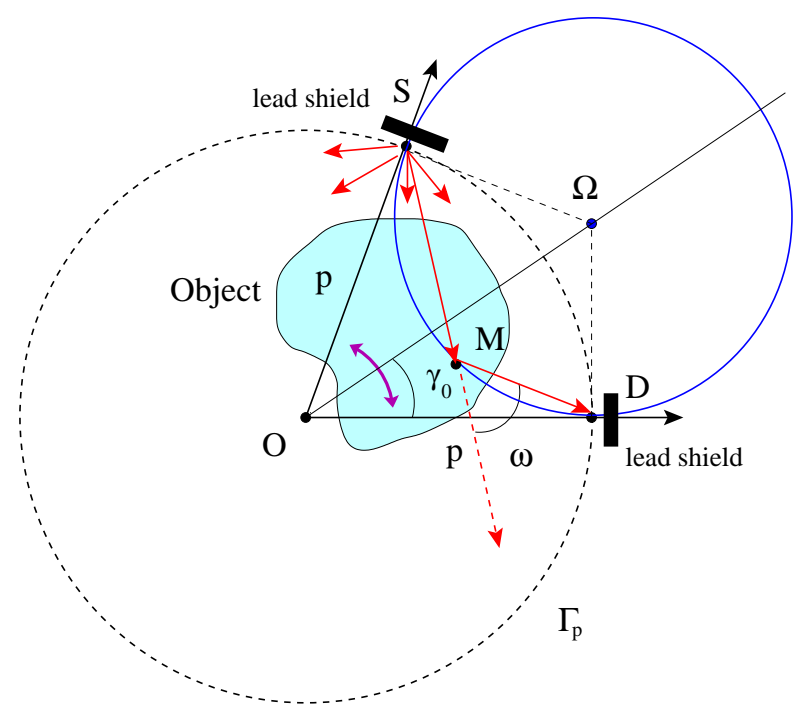

Figure 5. New Compton Scatter Tomography modality associated with internal circular $\operatorname{arcs} C_{p \tau}^{-}$

As natural extensions of this work, topics of future investigations could be for example:

- the inversion of Radon transforms on $\sigma$-descendants of the arcs of circle $C_{p \tau}^{ \pm}$, in particular when $\sigma=1 / m$ with $m=2,3, \ldots$ It is expected that relations to orthogonal function expansions arise as found in [3, 29],

- the search for manifolds of co-dimension 1 in $\mathbb{R}^{n}$, having a rotational symmetry axis going through the coordinate origin, on which Radon transforms can be defined and inverted exactly via harmonic component decomposition of functions. It is hoped that new innovating imaging principles could come up in $\mathbb{R}^{3}$.

\section{Acknowledgements}

The authors would like to thank the referees for their constructive criticism and suggestions, which have led to the improvement of this manuscript. They are also grateful to Professor E T Quinto for help to get the relevant literature on the circular Radon transform.

\section{References}

[1] Cormack A M 1963 Representation of a function by its line integrals, with some radiological applications J. Appl. Phys. 34, 2722-27; ibid. 196435 2908-12

[2] Cormack A M 1981 The Radon transform on a family of curves in the plane Proceedings of the American Mathematical Society 83(2), 325-30

[3] Cormack A M 1982 The Radon transform on a family of curves in the plane II Proceedings of the American Mathematical Society 86(2), 293-8

[4] Redding N J and Newsam G N 2001 Inverting the circular Radon Transform, DSTO Electronics and Surveillance Research Laboratory Report DSTO-RR-0211, Edinburgh, Australia. http://www.dsto.defence.gov.au/corporate/reports/DSTO-RR-0211.pdf 
[5] Sysoev S E 1997 Unique recovery of a function integrable in a strip from its integral over circles centered on a fixed line Communications of the Moscow Mathematical Society, 846-7

[6] Finch D, Patch S K and Rakesh 2004 Determining a function from its mean values over a family of spheres, SIAM J. Math. Anal 35(5), 1213-40

[7] Kurusa A 1992 The invertibility of the Radon transform on abstract totational manifold of real type Math. Scan.70, 112-6

[8] Kurusa A 1993 Support curves of invertible Radon transforms, Arch. Math., 61, 448-58

[9] Quinto E T 1983 The invertibility of rotational invariant Radon transforms J. Math. Anal. Appl. 90, $408-20$

[10] Quinto E T 1994 Radon transforms on curves in the plane Lectures in Applied Mathematics: Tomography, Impedance Imaging and Integral Geometry 30 231-44

[11] Globevnik J and Quinto E T 1996 Morerz theorems via microlocal analysis J. Geom. Analysis 6, 20-30

[12] Quinto E T 2001 Radon Transforms, Differential Equations and Microlocal Analysis Contemporary Mathematics, AMS 278, 57-68

[13] Perry M R 1975 Reconstructing a function by circular harmonic analysis of its Radon transform in Digest of topical meeting on Image Processing for 2-D and 3-D Reconstruction Opt. Soc. Am. Washington, DC 1975

[14] Verly J 1981 Circular and extended circular harmonic transforms and their relevance to image reconstruction from line integrals J. Opt. Soc. Am. 71(7), 825-35

[15] Li T 1960 A new class of integral transforms Proc. Amer. Math. Soc. 11, 290-8

[16] Nguyen M K and Truong T T 2010 Inversion of a new circular-arc Radon transform for Compton scattering tomography Inverse Problems 26, 065005

[17] Bushman R G Integrals of hypergeometric functions, Math. Zeitschr., 89, 74-6

[18] Yagle A E 1992 Inversion of spherical means using geometric inversion and Radon transforms Inverse Problems 8, 949-64

[19] Kurusa A 1993 The Radon transform on a half-sphere Acta Sci. Math. (Szeged) 58, 143-58

[20] Paladomov V P 2000 Reconstruction from limited data of arc means The journal of Fourier Analysis and Applications 6(1) 25-42

[21] Norton S J 1994, Compton scattering tomography, J. Appl. Phys. 76, 2007-15

[22] Cormack A M 1984 Radon's problem - Old and New, SIAM - AMS Proceedings 14, 33-9

[23] Magnus W, Oberhettinger F and Soni R P 1966 Formulas and Theorems for the Special Functions of Mathematical Physics 3rd Ed., Springer Verlag, Berlin.

[24] Agranovsky M and Quinto E T 1996 Injectivity sets for the Radon transform over circles and complete systems of radial functions J. Functional Analysis 139, 383-414

[25] Ambartsoumian G and Kuchment P 2005 On the injectivity of the circular Radon transform Inverse Problems 21, 473-85

[26] Ambartsoumian G and Kuchment P 2006 A range description for the planar circular Radon transform SIAM Journal on Mathematical Analysis 38(2), 681-92

[27] Agranovsky M and Kuchment P 2011 The support theorem for the single radius spherical mean transform Memoirs on Differential Equations and Mathematical Physics 52, 1-16

[28] Norton S J 1980 Reconstruction of a two-dimensional reflecting medium over a circular domain: exact solution, J. Acoust. Soc. Am. 64, 1266-73

[29] A.K. Louis 1984 Orthogonal function series expansions and the null space of the Radon transform, SIAM J. Math. Anal. 15, 621-33

[30] Barrett H H, The Radon Transform and its Applications in Progress in Optics, vol. 21, pp. 219-286, Ed. E. Wolf, North Holland (1984).

[31] Lale P G 1959 The examination of internal tissues, using gamma-ray scatter with a possible extension to megavoltage radiography, Phys. med. Biol. 4, 159-67

[32] Guzzardi R and Licitra G 1988 A critical review of Compton imaging, CRC Critical Reviews in Biomedical Imaging, 15(3), 237-68

[33] Kondic N N 1978 Density field determination by an external stationary radiation source using a kernel technique, in Measuremnts in Polyphase Flows Symposium ASME Winter Annual Meeting, San Francisco, California, Dec 10-15, 1978

[34] Prettyman T H 1991 Composition and density imaging of industrial samples by a combination of photon transmission tomography and projective Compton scatterometry, Ph.D Thesis, North Carolina State University

[35] Gerl J 2005 Gamma-ray imaging exploiting the Compton effect, Nuclear Physics A 752, 688-95c 